PROCEEDINGS OF THE

AMERICAN MATHEMATICAL SOCIETY

Volume 137, Number 10, October 2009, Pages 3421-3423

S 0002-9939(09)09924-9

Article electronically published on May 14, 2009

\title{
A NOTE ON TRANSITIVE LOCALIZING ALGEBRAS
}

\author{
MIGUEL LACRUZ
}

(Communicated by Marius Junge)

\begin{abstract}
A simple proof is provided for a theorem of Troitsky that every nonzero quasinilpotent operator on a Banach space whose commutant is a localizing algebra has a nontrivial hyperinvariant subspace.
\end{abstract}

The concept of a localizing algebra has been introduced recently by Lomonosov, Radjavi and Troitsky 10] as a condition to obtain invariant subspaces for operators on Banach spaces. A slightly stronger version of this notion had already been considered by Androulakis [1] as an assumption in the method of minimal vectors, a procedure to find invariant subspaces that was designed not long ago by Enflo 7 and that has been studied ever since then by many authors $1,2,2,3,4,5,6,8,10,12$.

Definition 1. Let $X$ be a Banach space and let $\mathcal{B}(X)$ denote the algebra of all bounded linear operators on $X$. A subalgebra $\mathcal{R}$ of $\mathcal{B}(X)$ is said to be localizing if there is a closed ball $B \subseteq X$ such that $0 \notin B$ and for every sequence $\left(x_{n}\right)$ in $B$ there is a subsequence $\left(x_{n_{j}}\right)$ and a sequence $\left(R_{j}\right)$ in $\mathcal{R}$ such that $\left\|R_{j}\right\| \leq 1$ and $\left(R_{j} x_{n_{j}}\right)$ converges in norm to a nonzero vector.

Recall that the commutant of a subset $\mathcal{S} \subseteq \mathcal{B}(X)$ is the algebra $\mathcal{S}^{\prime}$ of all operators that commute with every element of $\mathcal{S}$. A subspace $Y \subseteq X$ is said to be invariant under an operator $T \in \mathcal{B}(X)$ if $T Y \subseteq Y$. A subspace $Y \subseteq X$ is said to be invariant under a subalgebra $\mathcal{R} \subseteq \mathcal{B}(X)$ if $Y$ is invariant under every $R \in \mathcal{R}$. A subspace $Y \subseteq X$ is said to be hyperinvariant under an operator $T \in \mathcal{B}(X)$ if $Y$ is invariant under the subalgebra $\{T\}^{\prime}$. A subalgebra $\mathcal{R} \subseteq \mathcal{B}(X)$ is said to be transitive if the only closed subspaces invariant under $\mathcal{R}$ are the trivial ones, $Y=\{0\}$ and $Y=X$. It turns out that a subalgebra $\mathcal{R} \subseteq \mathcal{B}(X)$ is transitive if and only if for each nonzero vector $x \in X$, the orbit $\mathcal{R} x=\{R x: R \in \mathcal{R}\}$ is a dense subspace of $X$. Using the method of minimal vectors, Troitsky [12] obtained the following result.

Theorem 2. If $T$ is a nonzero quasinilpotent operator on a Banach space and $\{T\}^{\prime}$ is a localizing algebra, then $T$ has a nontrivial hyperinvariant subspace.

As pointed out by Lomonosov, Radjavi and Troitsky [10, the above result easily extends to algebras of operators as follows.

Theorem 3. If $\mathcal{R}$ is a transitive localizing subalgebra of $\mathcal{B}(X)$, then $\mathcal{R}^{\prime}$ does not contain any nonzero quasinilpotent operator.

Received by the editors August 7, 2008, and, in revised form, December 31, 2008, and February $13,2009$.

2000 Mathematics Subject Classification. Primary 47A15; Secondary 47L10.

Key words and phrases. Invariant subspace, localizing algebra, quasinilpotent operator.

This research was partially supported by Junta de Andalucía under Grant FQM-3737.

(C)2009 American Mathematical Society Reverts to public domain 28 years from publication 
The purpose of this paper is to provide a proof for this result that depends only on the spectral radius formula. This proof represents a simplification of the original one because it does not rely on the method of minimal vectors.

The first part of our proof is a claim isolated from the beginning of the proof for Theorem 2.3 in the paper of Lomonosov, Radjavi and Troitsky [10]. Its proof is included here for the sake of clarity and completeness.

The second part of our proof resembles an argument at the end of the proof in Hilden's simplification for the striking theorem of Lomonosov [9] that any nonzero compact operator on a complex Banach space has a nontrivial invariant subspace. We refer to the book of Rudin [11] for this argument.

Proof of Theorem [3. Let $T \in \mathcal{R}^{\prime}$ be a nonzero operator. We must show that $T$ is not quasinilpotent. Since $\mathcal{R}$ is transitive and $\operatorname{ker} T$ is a closed proper subspace of $X$ invariant under $\mathcal{R}$, it follows that $\operatorname{ker} T=\{0\}$, so that $T$ is injective.

Let $B \subseteq X$ be a ball as in Definition 1, We claim that there exists $c>0$ such that for every $x \in B$ there is an operator $R \in \mathcal{R}$ with $\|R\| \leq c$ and $R T x \in B$. If this is not so, then for every $n \geq 1$, there is a vector $x_{n} \in B$ such that $\|R\| \geq n$, whenever $R \in \mathcal{R}$ and $R T x_{n} \in B$. Since $\mathcal{R}$ is localizing, there is a subsequence $\left(x_{n_{j}}\right)$ and a sequence $\left(R_{j}\right)$ in $\mathcal{R}$ such that $\left\|R_{j}\right\| \leq 1$ and $\left(R_{j} x_{n_{j}}\right)$ converges in norm to some nonzero vector $x \in X$. We have $T R_{j}=R_{j} T$ for all $j \geq 1$, so that $\left(R_{j} T x_{n_{j}}\right)$ converges to $T x$ in norm. Now $T x \neq 0$ because $T$ is injective and $x \neq 0$. Since $\mathcal{R}$ is transitive, there is an operator $R \in \mathcal{R}$ such that $R T x \in \operatorname{int} B$. It follows that there is a $j_{0} \geq 1$ such that $R R_{j} T x_{n_{j}} \in \operatorname{int} B$ for every $j \geq j_{0}$. Since $R R_{j} \in \mathcal{R}$, the choice of the sequence $\left(x_{n}\right)$ implies that $\left\|R R_{j}\right\| \geq n_{j}$ for every $j \geq j_{0}$, and this is a contradiction because $\left\|R R_{j}\right\| \leq\|R\|$ for every $j \geq 1$.

Take a vector $x_{0} \in B$ and choose an operator $R_{1} \in \mathcal{R}$ with $\left\|R_{1}\right\| \leq c$ and such that $R_{1} T x_{0} \in B$. Now choose another operator $R_{2} \in \mathcal{R}$ with $\left\|R_{2}\right\| \leq c$ and such that $R_{2} T R_{1} T x_{0} \in B$. Continue this ping-pong game to obtain a sequence of vectors $\left(x_{n}\right)$ in $B$ and a sequence of operators $\left(R_{n}\right)$ in $\mathcal{R}$ such that $\left\|R_{n}\right\| \leq c$ and

$$
x_{n}=R_{n} T \cdots R_{1} T x_{0}=R_{n} \cdots R_{1} T^{n} x_{0} .
$$

Finally, let $d=\min \{\|x\|: x \in B\}$. It is plain that $d>0$ because $0 \notin B$. Hence,

$$
d \leq\left\|x_{n}\right\| \leq c^{n}\left\|T^{n}\right\| \cdot\left\|x_{0}\right\|,
$$

and this gives information on the spectral radius of $T$, namely,

$$
r(T)=\lim _{n \rightarrow \infty}\left\|T^{n}\right\|^{1 / n} \geq \frac{1}{c}>0 .
$$

This shows that $T$ fails to be quasinilpotent, as we wanted.

It was shown by Troitsky [12] that if a subalgebra $\mathcal{R}$ of $\mathcal{B}(X)$ contains a nonzero compact operator, then $\mathcal{R}$ is localizing. The following example goes to show that a localizing algebra may not contain nonzero compact operators. Let $\varphi \in C[0,1]$ and consider the multiplication operator $M_{\varphi}$ defined on $C[0,1]$ by the expression $\left(M_{\varphi} f\right)(t)=\varphi(t) f(t)$ for each $f \in C[0,1]$, so that $\left\|M_{\varphi}\right\|=\|\varphi\|_{\infty}$. Then consider the algebra of all multiplications $\mathcal{R}=\left\{M_{\varphi}: \varphi \in C[0,1]\right\}$. We claim that $\mathcal{R}$ is a localizing algebra. Consider the ball $B=\left\{f \in C[0,1]:\left\|f-\chi_{[0,1]}\right\|_{\infty} \leq 1 / 2\right\}$, so that $0 \notin B$. Now take any sequence $\left(f_{n}\right)$ in $B$ and notice that $\left|f_{n}(t)\right| \geq 1 / 2$ for each $t \in[0,1]$. Hence, $\varphi_{n}=1 /\left(2 f_{n}\right)$ is a continuous function with $\left\|\varphi_{n}\right\|_{\infty} \leq 1$, so that $M_{\varphi_{n}} \in \mathcal{R}$ and $\left\|M_{\varphi_{n}}\right\| \leq 1$. Moreover, the sequence $\left(M_{\varphi_{n}} f_{n}\right)$ converges in norm to a nonzero function because $M_{\varphi_{n}} f_{n} \equiv 1 / 2$ for every $n \geq 1$. We finally show 
that $\mathcal{R}$ does not contain nonzero compact operators. Let $M_{\varphi} \in \mathcal{R}$ be a nonzero multiplication, that is, $\varphi \neq 0$. Consider the sequence of functions $f_{n}(t)=\cos 2 \pi n t$ and notice that $\left\|f_{n}\right\|_{\infty} \leq 1$ but $\left(M_{\varphi} f_{n}\right)$ has no pointwise convergent subsequence. This shows that in fact the operator $M_{\varphi}$ fails to be weakly compact. We have been told by Luis Rodríguez-Piazza in a private communication that $M_{\varphi}$ fails to be strictly singular, that is, that there is an infinite-dimensional subspace such that the restriction of $M_{\varphi}$ to it is invertible.

\section{ACKNOWLEDGEMENTS}

We would like to thank Fernando León-Saavedra, Victor Lomonosov, and Luis Rodríguez-Piazza for carefully reading the manuscript. Also, we would like to thank the referee for providing helpful comments.

\section{REFERENCES}

1. George Androulakis, A note on the method of minimal vectors, Trends in Banach spaces and operator theory (Memphis, TN, 2001), Contemp. Math., vol. 321, Amer. Math. Soc., Providence, RI, 2003, pp. 29-36. MR1978805 (2005b:47014)

2. Razvan Anisca and Vladimir G. Troitsky, Minimal vectors of positive operators, Indiana Univ. Math. J. 54 (2005), no. 3, 861-872. MR2151236 (2006c:47041)

3. Shamim Ansari and Per Enflo, Extremal vectors and invariant subspaces, Trans. Amer. Math. Soc. 350 (1998), no. 2, 539-558. MR1407476 (98d:47019)

4. Isabelle Chalendar and Jonathan R. Partington, Convergence properties of minimal vectors for normal operators and weighted shifts, Proc. Amer. Math. Soc. 133 (2005), no. 2, 501-510 (electronic). MR2093074 (2006d:47015)

5. - Variations on Lomonosov's theorem via the technique of minimal vectors, Acta Sci. Math. (Szeged) 71 (2005), no. 3-4, 603-617. MR2206598 (2006m:47005)

6. Isabelle Chalendar, Jonathan R. Partington, and Martin Smith, Approximation in reflexive Banach spaces and applications to the invariant subspace problem, Proc. Amer. Math. Soc. 132 (2004), no. 4, 1133-1142 (electronic). MR2045430 (2005d:46023)

7. Per Enflo, Extremal vectors for a class of linear operators, Functional analysis and economic theory (Samos, 1996), Springer, Berlin, 1998, pp. 61-64. MR.1730119 (2000k:47020)

8. Hailegebriel E. Gessesse and Vladimir G. Troitsky, Invariant subspaces of positive quasinilpotent operators on ordered Banach spaces, Positivity 12 (2008), no. 2, 193-208. MR2398994 (2009c:47060)

9. V. I. Lomonosov, Invariant subspaces of the family of operators that commute with a completely continuous operator, Funkcional. Anal. i Priložen. 7 (1973), no. 3, 55-56. MR0420305 $(54: 8319)$

10. Victor I. Lomonosov, Heydar Radjavi, and Vladimir G. Troitsky, Sesquitransitive and localizing operator algebras, Integral Equations Operator Theory 60 (2008), no. 3, 405-418. MR2392834

11. Walter Rudin, Functional analysis, second ed., International Series in Pure and Applied Mathematics, McGraw-Hill, Inc., New York, 1991. MR.1157815 (92k:46001)

12. Vladimir G. Troitsky, Minimal vectors in arbitrary Banach spaces, Proc. Amer. Math. Soc. 132 (2004), no. 4, 1177-1180 (electronic). MR2045435(2005a:47010)

Departamento de Análisis Matemático, Facultad de Matemáticas, Universidad de Sevilla, Avenida Reina Mercedes s/n, 41012 Sevilla, Spain

E-mail address: lacruz@us.es 